\title{
SOME PROPERTIES OF COFINITELY WEAK ESSENTIAL SUPPLEMENTED MODULES
}

\author{
BERNA KOŞAR \\ Received 14 September, 2020
}

\begin{abstract}
Let $M$ be an $R$-module. If every cofinite essential submodule of $M$ has a weak supplement in $M$, then $M$ is called a cofinitely weak essential supplemented (or briefly cwe-supplemented) module. In this work, some properties of these modules are investigated. It is proved that any sum of cwe-supplemented modules is cwe-supplemented. It is also proved that every factor module and every homomorphic image of a cwe-supplemented module are cwe-supplemented.
\end{abstract}

2010 Mathematics Subject Classification: 16D10; 16D70

Keywords: Cofinite Submodules, Essential Submodules, Small Submodules, Supplemented Modules

\section{INTRODUCTION}

Throughout this paper all rings will be associative with identity and all modules will be unital left modules.

Let $R$ be a ring and $M$ be an $R$-module. We will denote a submodule $N$ of $M$ by $N \leq M$. Let $M$ be an $R$-module and $N \leq M$. If $L=M$ for every submodule $L$ of $M$ such that $M=N+L$, then $N$ is called a small (or superfluous) submodule of $M$ and denoted by $N \ll M$. A submodule $N$ of an $R$-module $M$ is called an essential submodule and denoted by $N \unlhd M$ in case $K \cap N \neq 0$ for every submodule $K \neq 0$, or equvalently, $N \cap L=0$ for $L \leq M$ implies that $L=0$. A submodule $K$ of $M$ is called a cofinite submodule of $M$ if $M / K$ is finitely generated. Let $M$ be an $R$-module and $U, V \leq M$. If $M=U+V$ and $V$ is minimal with respect to this property, or equivalently, $M=U+V$ and $U \cap V \ll V$, then $V$ is called a supplement of $U$ in $M$. $M$ is called a supplemented module if every submodule of $M$ has a supplement in $M$. $M$ is called an essential supplemented module if every essential submodule of $M$ has a supplement in $M . M$ is called a cofinitely supplemented module if every cofinite submodule of $M$ has a supplement in $M$. $M$ is called a cofinitely essential supplemented module if every cofinite essential submodule of $M$ has a supplement in $M$. Let $M$ be an $R$-module and $U \leq M$. If for every $V \leq M$ such that $M=U+V, U$ has a supplement $V^{\prime}$ with $V^{\prime} \leq V$, we say $U$ has ample supplements in $M$. If every 
submodule of $M$ has ample supplements in $M$, then $M$ is called an amply supplemented module. If every essential submodule of $M$ has ample supplements in $M$, then $M$ is called an amply essential supplemented module. If every cofinite submodule of $M$ has ample supplements in $M$, then $M$ is called an amply cofinitely supplemented module. If every cofinite essential submodule of $M$ has ample supplements in $M$, then $M$ is called an amply cofinitely essential supplemented module. Let $M$ be an $R$-module and $U, V \leq M$. If $M=U+V$ and $U \cap V \ll M$, then $V$ is called a weak supplement of $U$ in $M . M$ is said to be weakly supplemented if every submodule of $M$ has a weak supplement in $M . M$ is said to be cofinitely weak supplemented if every cofinite submodule of $M$ has a weak supplement in $M . M$ is called a weakly essential supplemented module if every essential submodule of $M$ has a weak supplement in $M$. The intersection of all maximal submodules of an $R$-module $M$ is called the $\mathrm{rad}$ ical of $M$ and denoted by $\operatorname{Rad} M$. If $M$ have no maximal submodules, then we denote $R a d M=M$. Let $M$ be an $R$-module and $U, K \leq M$. We say $U$ lies above $K$ in $M$ if $K \leq U$ and $U / K \ll M / K$.

More informations about (amply) supplemented modules are in [4, 12-14]. The definitions of (amply) essential supplemented modules and some properties of them are in $[8,10,11]$. The definitions of (amply) cofinitely supplemented modules and some properties of them are in [1]. The definitions of (amply) cofinitely essential supplemented modules and some details of them are in [6,7]. Some details about weakly supplemented and cofinitely weak supplemented modules are in $[2,4]$. The definition of weakly essential supplemented modules and some properties of these modules are in [9].

\section{COFINITELY WEAK ESSENTIAL SUPPLEMENTED MODULES}

Definition 1. Let $M$ be an $R$-module. If every cofinite essential submodule of $M$ has a weak supplement in $M$, then $M$ is called a cofinitely weak essential supplemented (or briefly cwe-supplemented) module. (See also [5])

Lemma 1. Let $M$ be a finitely generated $R$-module. Then $M$ is weakly essential supplemented if and only if $M$ is cwe-supplemented.

Proof. Clear from definitions.

Proposition 1. Let $M$ be a cwe-supplemented module. Then $M /$ RadM have no proper cofinite essential submodules.

Proof. Let $\frac{K}{\operatorname{Rad} M}$ be any cofinite essential submodule of $\frac{M}{\operatorname{Rad} M}$. By $\frac{M}{K} \cong \frac{M / \operatorname{Rad} M}{K / \operatorname{Rad} M}$, $K$ is a cofinite submodule of $M$. Since $\frac{K}{\operatorname{Rad} M} \unlhd \frac{M}{\operatorname{Rad} M}$, then $K \unlhd M$ and since $M$ is cwe-supplemented, $K$ has a weak supplement $V$ in $M$. Here $M=K+V$ and $K \cap V \ll$ $M$. Since $M=K+V, \frac{M}{\operatorname{Rad} M}=\frac{K}{\operatorname{Rad} M}+\frac{V+\operatorname{Rad} M}{\operatorname{Rad} M}$. Since $K \cap V \ll M$, by [13, 21.5], $K \cap V \leq \operatorname{Rad} M$. Then $\frac{K}{\operatorname{Rad} M} \cap \frac{V+\operatorname{Rad} M}{\operatorname{Rad} M}=\frac{K \cap V+\operatorname{Rad} M}{\operatorname{Rad} M}=0$ and $\frac{M}{\operatorname{Rad} M}=\frac{K}{\operatorname{Rad} M} \oplus \frac{V+\operatorname{Rad} M}{\operatorname{Rad} M}$. 
Since $\frac{M}{\operatorname{Rad} M}=\frac{K}{\operatorname{Rad} M} \oplus \frac{V+\operatorname{Rad} M}{\operatorname{Rad} M}$ and $\frac{K}{\operatorname{Rad} M} \unlhd \frac{M}{\operatorname{Rad} M}, \frac{K}{\operatorname{Rad} M}=\frac{M}{\operatorname{Rad} M}$. Hence $\frac{M}{\operatorname{Rad} M}$ have no proper cofinite essential submodules.

Proposition 2. Let $M$ be a cwe-supplemented module. If $K$ is a proper cofinite essential submodule of $M$ and Rad $M \leq K$, then $K /$ RadM is not essential in $M / R a d M$.

Proof. Since $\operatorname{Rad} M \leq K$ and $K \neq M, K / \operatorname{Rad} M \neq M / \operatorname{Rad} M$. Since $M$ is cwesupplemented, $K$ has a weak supplement $V$ in $M$. Here $M=K+V$ and $K \cap V \ll M$. Since $M=K+V, \frac{M}{\operatorname{Rad} M}=\frac{K}{\operatorname{Rad} M}+\frac{V+\operatorname{Rad} M}{\operatorname{Rad} M}$. By $K \cap V \leq \operatorname{Rad} M, \frac{K}{\operatorname{Rad} M} \cap \frac{V+\operatorname{Rad} M}{\operatorname{Rad} M}=$ $\frac{K \cap V+\operatorname{Rad} M}{\operatorname{Rad} M}=0$ and $\frac{M}{\operatorname{Rad} M}=\frac{K}{\operatorname{Rad} M} \oplus \frac{V+\operatorname{Rad} M}{\operatorname{Rad} M}$. Following these we have $\frac{V+\operatorname{Rad} M}{\operatorname{Rad} M} \neq 0$ and since $\frac{K}{\operatorname{Rad} M} \cap \frac{V+\operatorname{Rad} M}{\operatorname{Rad} M}=0, K / \operatorname{Rad} M$ is not essential in $M / \operatorname{Rad} M$.

Lemma 2. Let $M$ be an $R$-module, $U$ be a cofinite essential submodule of $M$ and $M_{1} \leq M$. If $M_{1}$ is cwe-supplemented and $U+M_{1}$ has a weak supplement in $M$, then $U$ has a weak supplement in $M$.

Proof. Let $X$ be a weak supplement of $U+M_{1}$ in $M$. Then $M=U+M_{1}+X$ and $X \cap\left(U+M_{1}\right) \ll M$. Since $U$ is a cofinite submodule of $M$ and $\frac{M / U}{(U+X) / U} \cong$ $\frac{M}{U+X}=\frac{M_{1}+U+X}{U+X} \cong \frac{M_{1}}{M_{1} \cap(U+X)}, M_{1} \cap(U+X)$ is a cofinite submodule of $M_{1}$. Since $U \unlhd M,(U+X) \unlhd M$ and $(U+X) \cap M_{1} \unlhd M_{1}$. Then by $M_{1}$ being cwe-supplemented, $(U+X) \cap M_{1}$ has a weak supplement $Y$ in $M_{1}$. Here $M_{1}=(U+X) \cap M_{1}+Y$ and $(U+X) \cap Y=(U+X) \cap M_{1} \cap Y \ll M_{1} \leq M$. Then $M=U+M_{1}+X=U+X+$ $(U+X) \cap M_{1}+Y=U+X+Y$ and $U \cap(X+Y) \leq(U+X) \cap Y+(U+Y) \cap X \leq$ $\left(U+M_{1}\right) \cap X+(U+X) \cap Y \ll M$. Hence $X+Y$ is a weak supplement of $U$ in $M$.

Corollary 1. Let $U$ be a cofinite essential submodule of $M$ and $M_{i} \leq M$ for $i=$ $1,2, \ldots, n$. If $M_{i}$ is cwe-supplemented for every $i=1,2, \ldots, n$ and $U+M_{1}+M_{2}+\ldots+$ $M_{n}$ has a weak supplement in $M$, then $U$ has a weak supplement in $M$.

Proof. Clear from Lemma 2.

Lemma 3. Any sum of cwe-supplemented modules is cwe-supplemented.

Proof. Let $U$ be a cofinite essential submodule of $M$ and $M=\sum_{\lambda \in \Lambda} M_{\lambda}$ for $M_{\lambda} \leq M$ and $M_{\lambda}$ be cwe-supplemented for every $\lambda \in \Lambda$. Since $U$ is a cofinite submodule of $M$, then there exist $\lambda_{1}, \lambda_{2}, \ldots, \lambda_{n} \in \Lambda$ such that $M=U+M_{\lambda_{1}}+M_{\lambda_{2}}+\ldots+M_{\lambda_{n}}$. Then 0 is a weak supplement of $U+M_{\lambda_{1}}+M_{\lambda_{2}}+\ldots+M_{\lambda_{n}}$ in $M$. Since $M_{\lambda_{i}}$ is cwe-supplemented for every $i=1,2, \ldots, n$, by Corollary $1, U$ has a weak supplement in $M$. Hence $M$ is cwe-supplemented.

Corollary 2. Let $M$ be a cwe-supplemented $R$-module. Then $M^{(\Lambda)}$ is cwe-supplemented for every index set $\Lambda$.

Proof. Clear from Lemma 3.

Lemma 4. Every factor module of a cwe-supplemented module is cwe-supplemented. 
Proof. Let $M$ be a cwe-supplemented $R$-module and $\frac{M}{K}$ be any factor module of $M$. Let $\frac{U}{K}$ be a cofinite essential submodule of $\frac{M}{K}$. Then $U$ is a cofinite essential submodule of $M$ and since $M$ is cwe-supplemented, $U$ has a weak supplement $V$ in $M$. Here $M=U+V$ and $U \cap V \ll M$. Following we have $\frac{M}{K}=\frac{U}{K}+\frac{V+K}{K}$ and $\frac{U}{K} \cap \frac{V+K}{K}=\frac{U \cap V+K}{K} \ll \frac{V+K}{K}$. Hence $\frac{V+K}{K}$ is a weak supplement of $\frac{U}{K}$ in $\frac{M}{K}$ and $\frac{M}{K}$ is cwe-supplemented.

Corollary 3. Every homomorphic image of a cwe-supplemented module is cwesupplemented.

Proof. Clear from Lemma 4.

Lemma 5. Let $M$ be a cwe-supplemented module. Then every $M$-generated $R$-module is cwe-supplemented.

Proof. Let $N$ be a $M$-generated $R$-module. Then there exist an index set $\Lambda$ and an $R$-module epimorphism $f: M^{(\Lambda)} \longrightarrow N$. Since $M$ is cwe-supplemented, by Corollary 2, $M^{(\Lambda)}$ is cwe-supplemented. Then by Corollary 3, $N$ is cwe-supplemented.

Lemma 6. Let $M$ be an $R$-module, $K \ll M$ and $\frac{U+K}{K} \unlhd \frac{M}{K}$ for every $U \unlhd M$. If $M / K$ is cwe-supplemented, then $M$ is also cwe-supplemented.

Proof. Let $U$ be any cofinite essential submodule of $M$. Since $U$ is a cofinite submodule of $M$, we clearly see that $U+K$ is a cofinite submodule of $M$. By $\frac{M / K}{(U+K) / K} \cong$ $\frac{M}{U+K},(U+K) / K$ is a cofinite submodule of $M / K$. By hypothesis, $\frac{U+K}{K} \unlhd \frac{M}{K}$ and since $M / K$ is cwe-supplemented, $\frac{U+K}{K}$ has a weak supplement $V / K$ in $M / K$. Here $\frac{M}{K}=\frac{U+K}{K}+\frac{V}{K}=\frac{U+V}{K}$ and $\frac{U \cap V+K}{K}=\frac{U+K}{K} \cap \frac{V}{K} \ll \frac{M}{K}$. Since $\frac{M}{K}=\frac{U+V}{K}$, then $M=U+V$. Let $U \cap V+T=M$ with $T \leq M$. Then $\frac{U \cap V+K}{K}+\frac{T+K}{K}=\frac{M}{K}$ and since $\frac{U \cap V+K}{K} \ll \frac{M}{K}$, $\frac{T+K}{K}=\frac{M}{K}$ and we have $T+K=M$. Since $K \ll M$, we have $T=M$. Hence $U \cap V \ll M$ and $V$ is a weak supplement of $U$ in $M$. Therefore, $M$ is cwe-supplemented.

Corollary 4. Let $f: M \longrightarrow N$ be an $R$-module epimorphism, $\operatorname{Ker}(f) \ll M$ and $f(U) \unlhd N$ for every $U \unlhd M$. If $N$ is cwe-supplemented, then $M$ is also cwe-supplemented.

Proof. Clear from Lemma 6.

Proposition 3. Let $R$ be a ring. The following assertions are equivalent.

$(i)_{R} R$ is weakly essential supplemented

(ii) ${ }_{R} R$ is cwe-supplemented.

(iii) $R^{(\Lambda)}$ is cwe-supplemented for every index set $\Lambda$.

(iv) Every $R$-module is cwe-supplemented.

Proof. $(i) \Longleftrightarrow$ (ii) Clear from Lemma 1 , since ${ }_{R} R$ is finitely generated.

(ii) $\Longleftrightarrow($ iii $)$ Clear from Corollary 2. 
(iii) $\Longrightarrow($ iv) Let $M$ be an $R$-module. Then there exist an index set $\Lambda$ and an $R$-module epimorphism $f: R^{(\Lambda)} \longrightarrow M$. Since $R^{(\Lambda)}$ is cwe-supplemented, by Corollary $3, M$ is also cwe-supplemented.

(iv) $\Longrightarrow($ ii) Clear.

Let $M$ be an $R$-module. We say submodules $X$ and $Y$ of $M$ are $\beta^{*}$ equivalent, $X \beta^{*} Y$, if and only if $(X+Y) / X \ll M / X$ and $(X+Y) / Y \ll M / Y$. More details about $\beta^{*}$ relation are in [3].

Lemma 7. Let $M$ be an $R$-module. If every cofinite essential submodule of $M$ is $\beta^{*}$ equivalent to a weak supplement submodule in $M$, then $M$ is cwe-supplemented.

Proof. Let $U$ be any cofinite essential submodule of $M$. By hypothesis, there exists a weak supplement submodule $X$ such that $U \beta^{*} X$ in $M$. Since $X$ is a weak supplement submodule in $M$, there exists $V \leq M$ such that $X$ is a weak supplement of $V$ in $M$. Then $V$ is a weak supplement of $X$ in $M$. Since $U \beta^{*} X$, by [3, Theorem 2.6 (ii)], $V$ is a weak supplement of $U$ in $M$. Hence $M$ is cwe-supplemented.

Corollary 5. Let $M$ be an $R$-module. If every cofinite essential submodule lies above a weak supplement submodule in $M$, then $M$ is cwe-supplemented.

Proof. Let $U$ be any cofinite essential submodule of $M$. By hypothesis, there exists a weak supplement submodule $X$ such that $U$ lies above $X$ in $M$. Since $U$ lies above $X$ in $M$, we clearly see that $U \beta^{*} X$ in $M$. Hence every cofinite essential submodule of $M$ is $\beta^{*}$ equivalent to a weak supplement submodule in $M$ and by Lemma 7, $M$ is cwe-supplemented.

Corollary 6. Let $M$ be an $R$-module. If every cofinite essential submodule of $M$ is $\beta^{*}$ equivalent to a supplement submodule in $M$, then $M$ is cwe-supplemented.

Proof. Clear from Lemma 7, since every supplement submodule is a weak supplement submodule in $M$.

Corollary 7. Let $M$ be an $R$-module. If every cofinite essential submodule lies above a supplement submodule in $M$, then $M$ is cwe-supplemented.

Proof. Clear from Corollary 5, since every supplement submodule is a weak supplement submodule in $M$.

Lemma 8. Let $M$ be a cwe-supplemented $R$-module. If every weak supplement of any cofinite essential submodule of $M$ is a supplement in $M$, then $M$ is cofinitely essential supplemented.

Proof. Let $U$ be any cofinite essential submodule of $M$. Since $M$ is cwe-supplemented, $U$ has a weak supplement $V$ in $M$. Here $M=U+V$ and $U \cap V \ll M$. By hypothesis, $V$ is a supplement in $M$. Since $U \cap V \ll M$, by [12, Lemma 2.5], $U \cap V \ll V$. Hence $V$ is a supplement of $U$ in $M$. Therefore, $M$ is cofinitely essential supplemented. 
Corollary 8. Let $M$ be a finitely generated cwe-supplemented $R$-module. If every weak supplement submodule in $M$ is a supplement in $M$, then $M$ is essential supplemented.

Proof. Clear from Lemma 8, since every submodule of $M$ is cofinite.

\section{REFERENCES}

[1] R. Alizade, G. Bilhan, and P. F. Smith, "Modules whose maximal submodules have supplements," Communications in Algebra, vol. 29, no. 6, pp. 2389-2405, 2001.

[2] R. Alizade and E. Büyükaşı, "Cofinitely weak supplemented modules," Comm. Algebra, vol. 31, no. 11, pp. 5377-5390, 2003, doi: 10.1081/AGB-120023962. [Online]. Available: https://doi.org/10.1081/AGB-120023962

[3] G. F. Birkenmeier, F. Takil Mutlu, C. Nebiyev, N. Sokmez, and A. Tercan, "Goldie*-supplemented modules," Glasg. Math. J., vol. 52, no. A, pp. 41-52, 2010, doi: 10.1017/S0017089510000212. [Online]. Available: https://doi.org/10.1017/S0017089510000212

[4] J. Clark, C. Lomp, N. Vanaja, and R. Wisbauer, Lifting Modules: Supplements and Projectivity in Module Theory (Frontiers in Mathematics), 2006th ed. Basel: Birkhäuser, 82006.

[5] B. Koşar, "Cofinitely weak e-supplemented modules," in 3rd International E-Conference on Mathematical Advances and Applications (ICOMAA-2020), 2020.

[6] B. Koşar and C. Nebiyev, "Cofinitely essential supplemented modules," Turkish Studies Information Technologies and Applied Sciences, vol. 13, no. 29, pp. 83-88, 2018.

[7] B. Koşar and C. Nebiyev, "Amply cofinitely essential supplemented modules," Archives of Current Research International, vol. 19, no. 1, pp. 1-4, 2019.

[8] C. Nebiyev, "E-supplemented modules," in Antalya Algebra Days, ser. XVIII, Şirince, İzmir, Turkey, 2016.

[9] C. Nebiyev and B. Koşar, "Weakly essential supplemented modules," Turkish Studies Information Technologies and Applied Sciences, vol. 13, no. 29, pp. 89-94, 2018.

[10] C. Nebiyev, H. H. Ökten, and A. Pekin, "Amply essential supplemented modules," Journal of Scientific Research and Reports, vol. 24, no. 4, pp. 1-4, 2018.

[11] C. Nebiyev, H. H. Ökten, and A. Pekin, "Essential supplemented modules," International Journal of Pure and Applied Mathematics, vol. 120, no. 2, pp. 253-257, 2018.

[12] C. Nebiyev and A. Pancar, "On supplement submodules," Ukrainian Math. J., vol. 65, no. 7, pp. 1071-1078, 2013, doi: 10.1007/s11253-013-0842-2. [Online]. Available: https://doi.org/10.1007/s11253-013-0842-2

[13] R. Wisbauer, Foundations of module and ring theory, german ed., ser. Algebra, Logic and Applications. Gordon and Breach Science Publishers, Philadelphia, PA, 1991, vol. 3, a handbook for study and research.

[14] H. Zöschinger, "Komplementierte Moduln über Dedekindringen," J. Algebra, vol. 29, pp. 42-56, 1974, doi: 10.1016/0021-8693(74)90109-4. [Online]. Available: https://doi.org/10.1016/ 0021-8693(74)90109-4

Author's address

Berna Koşar

Uskudar University, Department of Health Management, Üsküdar, İstanbul, Turkey

E-mail address: bernak@omu.edu.tr, berna.kosar@uskudar.edu.tr 